\title{
Kurzviten der Autorinnen und Autoren
}

Theodor Dieter Jg. 1951, studierte Philosophie (M.A.) und Evangelische Theologie in Heidelberg und Tübingen; Dr. theol. habil., Dres. h.c., 1984-2019 Forschungsprofessor am Institut für Ökumenische Forschung in Strasbourg und dessen Direktor von 1998 bis 2018; seit 2019 dort Senior Research Professor. Ratzinger-Preis 2017. Forschungsschwerpunkte: Luthers Theologie, insbesondere im Zusammenhang mit dem Mittelalter, Luthertum, Ökumenische Theologie.

Irene Dingel Jg. 1956, Direktorin des Leibniz-Instituts für Europäische Geschichte Mainz (IEG), Abt. für Abendländische Religionsgeschichte. Studium der evangelischen Theologie und der Romanistik an der Ruprecht-Karls-Universität Heidelberg und an der Faculté libre de Théologie protestante Paris sowie an der Sorbonne/Paris IV. Promotion (französische Sprachwissenschaft 1986, Dr. phil.) und Habilitation 1993 (Historische Theologie, Dr. phil. habil. theol.) an der Ruprecht-Karls-Universität Heidelberg. 1994-1998 Professorin für Historische Theologie an der Goethe-Universität Frankfurt/M. Seit 1998 Professorin für Kirchen- und Dogmengeschichte an der Johannes Gutenberg-Universität Mainz. Seit 2005 Direktorin des Leibniz-Instituts für Europäische Geschichte Mainz (= außeruniversitäres Forschungsinstitut, Mitglied der Leibniz-Gemeinschaft). Forschungsschwerpunkte: Reformation und Konfessionalisierung; Frühaufklärung in ihrem westeuropäischen Zusammenhang; Die Kirchen und Europa (20. Jahrhundert); Leitung der Langzeit-Forschungsprojekte „Controversia et Confessio“ und „Religiöse Friedenswahrung und Friedensstiftung in Europa. 1500-1800 - Europäische Religionsfrieden Digital“ bei der Akademie der Wissenschaften und der Literatur Mainz.

Johannes Ehmann Jg. 1958, Studium der evangelischen Theologie in Berlin, Jerusalem, Göttingen, Tübingen und Heidelberg, Pfarrer und Dr. theol., Geschäftsführer der ACK in Baden-Württemberg, heute apl. Prof. (hauptberuflich) für Kirchengeschichte an der Theologischen Fakultät der Universität Heidelberg.

Eva-Maria Faber Jg. 1964, Dr. theol. habil., Studium der katholischen Theologie in Münster, Toulouse und Freiburg i.B., 1992 Promotion, 1998 Habilitation. Seit 2000 Professorin für Dogmatik und Fundamentaltheologie an der Theologischen Hochschule Chur. Seit 2002 Konsultorin des Päpstlichen Rates zur Förderung der Einheit der Christen; seit 2009 Beraterin der Internationalen Lutherisch/römisch-katholischen Kommission für die Einheit. Arbeits- und Forschungsschwerpunkte: Ökumene, Theologie des 20. Jahrhunderts (Erich Przywara), Johannes Calvin.

Günter Frank Jg. 1956, apl. Professor für Philosophie am Karlsruher Institut für Technologie, geb. in Arnstadt/Thüringen, Studium von Theologie, Psychologie und Philosophie in Warschau, Erfurt und Vallendar/Rhein; 1986-1991 Assistent am philosophischen Lehrstuhl des Philosophisch-Theologischen Studiums in Erfurt; 1994 Promotion an der päpstlichen Universität Gregoriana; 1993-1995 fellowship der University of Chicago, Studium der Religionswissenschaft und Religionsphilosophie, weitere Forschungsaufenthalte an der Herzog August Bibliothek in Wolfenbüttel und am Warburg-Institute der University of London. Forschungsstipendien des Deutschen Akademischen Austauschdienstes (DAAD) und der Deutschen Forschungsgemeinschaft. Zwischen 1997 Lehrbeauftragter und seit 2001 Privatdozent für Philosophie an der Frei- 
en Universität in Berlin, seit 1998 Kustos des Melanchthonhauses in Bretten, seit 2004 Direktor der Europäischen Melanchthon-Akademie Bretten. Seit 2013 Lehrtätigkeit am Institut für Philosophie des Karlsruher Instituts für Technologie (KIT). Verschiedene Veröffentlichungen zur Philosophie und Theologie des Mittelalters, des Humanismus, der Reformation und der frühen Neuzeit.

Bernd Jochen Hilberath Jg. 1948, Dr. theol., 1967-1972 Studium der Philosophie und der Theologie in München und Mainz, 1977 Promotion, 1984 Habilitation für Dogmatische und Ökumenische Theologie, Prof. in Mainz, seit 1992 in Tübingen (Nachfolge Walter Kasper), seit 1996 auch Direktor des Instituts für Ökumenische Forschung (Nachfolge Hans Küng); 1986 Karl-Rahner-Preis für Theologische Forschung, 1987-1992 Mitglied des Deutschen Ökumenischen Studienausschusses, Vorstandsmitglied und 2004-2006 Präsident der societas oecumenica; 2013 Emeritierung.

Bo Kristian Holm Jg. 1970. PhD in Theologie, Aarhus 2001. Studium der Theologie in Aarhus und Tübingen. Forschungsschwerpunkte: Luther und Melanchthon, Gabe-Theologie, Lutherrenaissance, Regin Prenter, Sakramentstheologie, Konfessionskultur. Professor der Dogmatik an der Universität Aarhus, Leiter von LUMEN: Center for the Study of Lutheran Theology and Confessional Societies und Leiter des theologischen Forschungsprogramms an der Universität Aarhus.

Pascale Jung Jg. 1968, studierte Katholische Theologie in Tübingen und Dublin. Sie promovierte 2018 über den Begriff der Anerkennung in der Ökumene. Ihr Forschungsschwerpunkt liegt im Feld der Ökumene und in der Bewegung des „New Monasticism“. Sie ist Mitglied im Forschungskreis Kommunikative Theologie. Pascale Jung arbeitet als Pastoralreferentin im Bistum Trier und ist Vorsitzende der ACK Saarbrücken und Umgebung.

Kurt Kardinal Koch Jg. 1950, Prof. Dr. theol.; Priesterweihe 1982; Studien in Luzern und München und Promotion zum Doktor der Theologie. Er übernahm 1989 an der Theologischen Fakultät der Universität Luzern den Lehrstuhl für Dogmatik und Liturgiewissenschaft und lehrte am Katechetischen Institut ökumenische Theologie. 1995 wurde er zum Bischof von Basel gewählt, 1996 in Rom von Papst Johannes Paul II. zum Bischof geweiht. 1998 bis 2006 war er Vizepräsident, 2007 bis 2009 Präsident der Schweizer Bischofskonferenz. Papst Benedikt XVI. ernannte inn 2010 zum Erzbischof und Präsidenten des Päpstlichen Rates zur Förderung der Einheit der Christen. Im November 2010 kreierte und verkündete er ihn als Kardinal. Er war Diakon der Kirche Nostra Signora del Sacro Cuore di Gesù. Im Mai 2021 ernannte Papst Franziskus ihn zum Kardinalpriester; die bisherige Diakonie wurde pro hac vice zur Titelkirche erhoben.

Volker Leppin Jg. 1966, 1985-1991 Studium der Theologie und Germanistik in Marburg, Jerusalem und Heidelberg. 1994 Promotion, 1997 Habilitation, beides in Heidelberg; 1998-2000 Lehrstuhlvertretung Frankfurt a. M. 2000 - 2010 Professor für Kirchengeschichte in Jena, 2010 2021 in Tübingen, seit 2021 Horace Tracy Pitkin Professor of Historical Theology an der Yale Divinity School. Korr. Mitglied der Heidelberger und der Sächsischen Akademie der Wissenschaften. Forschungsschwerpunkte in Scholastik und Frömmigkeitsgeschichte des Mittelalters sowie in der Reformation. 
Tobias Licht Jg. 1962, 1981-1986 Studium der kath. Theologie in Freiburg i.B., Rom (Gregoriana) und Frankfurt a.M. (St. Georgen), Dipl.-Theol., 1994-2004 Wiss. Mitarb./Pers. Ref. des Weihbischofs und Diözesanadministrators von Freiburg, seit 2004 Leiter des Bildungszentrums - heute Bildungszentrum Roncalli-Forum - Karlsruhe (Bildungswerk der Erzdiözese Freiburg), zugleich Initiator und 2007-2019 kath. Leiter des Karlsruher Foyers Kirche und Recht (Kontaktstelle der Erzdiözese Freiburg und der Evangelischen Landeskirche in Baden zu den obersten Gerichten des Bundes in Karlsruhe), Publikationen zu systematisch-theologischen und kirchlichen Fragen.

Burkhard Neumann Jg. 1961, Studium der Philosophie und Theologie in Paderborn und Freiburg/Schweiz; Promotion 1996, Habilitation 2009; seit 2004 Direktor am Johann-Adam-Möhler-Institut für Ökumenik in Paderborn; Forschungs- und Veröffentlichungsschwerpunkte: Ökumenische Theologie, Sakramentenlehre, Ekklesiologie, Eschatologie.

Peter Neuner Jg. 1941, Studium katholische Theologie in München, Kaplan in Traunstein (1966 -1968). Promotion (1976) und Habilitation (1978) in München. 1980-1985 Professor für Fundamentaltheologie in Passau, 1985- 2006 Professor für Dogmatik und ökumenische Theologie, Universität München. Sprecher des Zentrums für Ökumenische Theologie, Universität München. 2006 Emeritierung. Zahlreiche Publikationen zum katholischen Modernismus, zur Ekklesiologie und zur Ökumene.

Friederike Nüssel Jg. 1961, hat evangelische Theologie und Religionsphilosophie in Tübingen, Göttingen, London und München studiert. Sie wurde 1994 in München promoviert und habilitierte sich dort 1998. 2001 wurde sie Professorin für Systematische Theologie und Direktorin des Ökumenischen Instituts an der Evangelisch-theologischen Fakultät der Universität Münster. Seit 2006 ist sie Professorin für Systematische Theologie und Direktorin des Ökumenischen Instituts an der Universität Heidelberg. Zu ihren Forschungsgebieten gehören die Entwicklung der Dogmatik in der Neuzeit, insbesondere Christologie, Rechtfertigungslehre und Ekklesiologie, die Herausforderungen und Potentiale konfessioneller Differenzierung, Dialoge und Einheitsvorstellungen in der Ökumene und interdisziplinäre Fragestellungen im Bereich der Anthropologie.

Johanna Rahner Jg. 1962, Studium der Theologie und Biologie an der Universität Freiburg; 2006 - 2010 Professorin für Dogmatik an der Kath.-theol. Fakultät der Otto-Friedrich-Universität Bamberg; 2010 - 2014 Professorin für Systematische Theologie am Institut für Katholische Theologie der Universität Kassel; seit April 2014 Professorin für Dogmatik, Dogmengeschichte und Ökumene an der Kath.-theol. Fakultät der Eberhard-Karls Universität Tübingen und Direktorin des dortigen Instituts für Ökumenische und Interreligiöse Forschung.

Risto Saarinen Jg. 1959, Dr. theol. 1988, Dr. phil. 1994, Dr. h.c. 2017 (Kopenhagen), studierte Theologie und Philosophie in Helsinki, Forschungsschwerpunkte: Reformation, Geschichte der Philosophie, Ökumenische Theologie, seit 2001 Professor für Ökumenische Theologie an der Universität Helsinki.

Dorothea Sattler Jg. 1961, Dr. theol., Studium der kath. Theologie und Romanistik in Freiburg und Mainz. 1992 Promotion und 1996 Habilitation in Mainz. 1996-1998 Gastprofessorin an der Freien Universität in Berlin sowie 1998-2000 Professorin für Systematische Theologie und 
Religionspädagogik in Wuppertal. Seit 2000 Professorin für Dogmatik und Ökumenische Theologie an der Katholisch-Theologischen Fakultät der Universität Münster sowie Direktorin des dortigen Ökumenischen Instituts. Zu den Hauptgebieten ihrer Forschungen zählen: Ökumenische Theologie, besonders in Bezug auf das menschliche Bußwerk sowie die Eucharistielehre; Soteriologie.

Herman J. Selderhuis Jg. 1961, ist Rektor und Professor für Kirchengeschichte an der Theologischen Universität Apeldoorn, Direktor von Refo500, sowie Präsident von RefoRC (Reformation Research Consortium).

Notger Slenczka Jg. 1960, Studium Philosophie und Theologie in Tübingen, München und Göttingen, Promotion (1990), Ordination (1996) und Habilitation (1997) in Göttingen. Lehrstuhlvertretungen in Mainz, Gießen und Tübingen. Lehrstuhl für Systematische Theologie und Sozialethik in Mainz 2000-2006, seit 2006 Lehrstuhl für Systematische Theologie (Dogmatik) an der HU zu Berlin. Geschäftsführender Universitätsprediger.

Hendrik Stössel Jg. 1953, Studium der Rechtswissenschaften in Freiburg i.B.; 1978 Erste Juristische Staatsprüfung; 1980 Zweite Juristische Staatsprüfung; Studium der Evang. Theologie in Heidelberg; 1985 Erste Theologische Prüfung, 1987 Zweite Theologische Prüfung und Pfarrvikariat; 1989 Gemeindepfarrer in Emmendingen; 1995 kirchenrechtliche Promotion zum Dr. theol.; 1998 Dekan und Gemeindepfarrer in Pforzheim; seit Oktober 2012 Theologischer Referent der Evangelischen Landeskirche in Baden an der Europäischen Melanchthon-Akademie Bretten.

Wolfgang Thönissen Jg. 1955, Studium der Katholischen Theologie und der Philosophie in Bonn und Tübingen, Promotion 1986 in Tübingen, Habilitation 1994 in Freiburg, seit 1999 Professor der Ökumenischen Theologie an der Theologischen Fakultät Paderborn und Leitender Direktor des Johann-Adam-Möhler-Instituts für Ökumenik in Paderborn, Konsultor des Päpstlichen Rates zur Förderung der Einheit der Christen, zahlreiche Veröffentlichungen zu Fragen der ökumenischen Hermeneutik, der Kirchengemeinschaft und der katholischen Lutherforschung.

Klaus Unterburger Jg. 1971, Dr. theol., habil., Dr. theol., Lic. theol., M.A., studierte Katholische Theologie und Philosophie in einem Doppelstudium und Evangelische Theologie im Nebenfach in München. Zu seinen Forschungs- und Veröffentlichungsschwerpunkten gehören die frühneuzeitliche Kirchengeschichte, die Theologiegeschichte seit dem Mittelalter und die Kirchengeschichte des 19. und 20. Jahrhunderts, seit 2012 ist er Inhaber des Lehrstuhls für mittlere und neue Kirchengeschichte an der Fakultät für katholische Theologie der Universität Regensburg.

Timothy J. Wengert Jg. 1950, Neben einem Bakkalaureat in Soziologie (1972) und einem Magistergrad (1973) in Geschichte der Universität Michigan hat Prof. Dr. Wengert auch einen Magistergrad in Theologie (Master of Divinity; 1977) des Luther Seminary (St. Paul, Minnesota) und einen Doktorgrad in Religion der Duke Universität (Durham, North Carolina; 1984). Sein Hauptinteresse gilt der Geschichte der biblischen Exegese, vor allem bei Luther und Melanchthon, und auch im Allgemeinen der Theologie der Wittenberger und der lutherischen Bekenntnisschriften. Er war Teilnehmer an verschiedenen ökumenischen Dialogen. Seit 2013 ist er im Ruhestand und ist „the Ministerium of Pennsylvania Professor emeritus of Reformation History at the United Lutheran Seminary [vormals The Lutheran Theological Seminary at Philadelphia].“ 
Gunther Wenz Jg. 1949, Prof. Dr. Dr. h.c., war Ordinarius für Systematische Theologie an der Evangelisch-Theologischen Fakultät der Ludwig-Maximilians-Universität München und leitet derzeit die Pannenberg-Forschungsstelle an der Münchener Hochschule für Philosophie. Der Schwerpunkt seiner Forschungen liegt im Bereich der Dogmatik und der Religionsphilosophie.

Christian V. Witt Jg. 1980, PD Dr. theol. habil., ist Heisenberg-Stipendiat der Deutschen Forschungsgemeinschaft am Leibniz-Institut für Europäische Geschichte Mainz; Studium der Evangelischen Theologie, Geschichtswissenschaften und Erziehungswissenschaften in Wuppertal; 2010 Promotion und 2015 Habilitation im Fach Kirchengeschichte; 2006 Erstes und 2011 Zweites Staatsexamen für das Gymnasiallehramt; Forschungsschwerpunkte in der Kirchen- und Theologiegeschichte des Mittelalters und der Neuzeit. 
